\title{
SOME NOVEL INFINITE SERIES OF SPHERICAL BESSEL FUNCTIONS*
}

\author{
BY \\ ANDREW N. VAVRECK (The Johns Hopkins University) \\ AND \\ WILLIAM THOMPSON, JR. (The Pennsylvania State Univeristy)
}

\begin{abstract}
By a double application of the translational additional theorem for spherical wave functions, whereby one shifts an $n$th order axisymmetric wave function from some origin to another and then in turn back to the first, one obtains a mathematical identity in the form of the $n$th order spherical wave function equated to an infinite series containing every order spherical wave function. The coefficients of the terms in this infinite series are themselves infinite series of spherical Bessel functions of arbitrary argument. These latter series must either sum to zero or unity to satisfy the mathematical identity. Following this reasoning, a collection of infinite series involving spherical Bessel functions has been generated. Some of the low mode order results are presented.
\end{abstract}

In a spherical coordinate system $(r, \theta, \phi)$, centered at point 0 , let the point $\left(r_{0}, 0,0\right)$ be the origin $0^{\prime}$ of a second spherical coordinate system $\left(r \cdot \theta^{\prime}, \phi\right)$. It has been shown, [1], that the $n$th order, axisymmetric, spherical wave function, of wave number $k$, centered at origin 0 , can be expressed as an infinite series of spherical wave functions centered at $0^{\prime}$ in the following form

$$
\begin{array}{r}
h_{n}(k r) P_{n}(\cos -)=\sum_{\nu=0}^{\infty} \sum_{p} i^{\nu+p-n}(2 \nu+1) a(0,0 \mid p, \nu, n) j_{\nu}\left(k r_{0}\right) \\
\cdot h_{p}\left(k r^{\prime}\right) P_{p}\left(\cos \theta^{\prime}\right), \text { for } r_{0}<r^{\prime} .
\end{array}
$$

This equation, the translation addition theorem for spherical wave functions, is valid for spherical Hankel functions of either kind. The index $p$ ranges over a finite set of values given by

$$
p=n+\nu, n+\nu-2, \ldots,|n-\nu| .
$$

The coefficients $a(0,0 \mid p, \nu, n)$ have been shown [2] to be expressible in the form

$$
\begin{array}{r}
a(0,0 \mid p, \nu, n)=(2 p+1)\left[\frac{(\nu+p-n) !(n+p-\nu) !(n+\nu-p) !}{(n+\nu+p+1) !}\right] \\
\cdot\left[\frac{(n+\nu+p / 2) !}{(\nu+p-n / 2) !(n+p-\nu / 2) !(n+\nu-p / 2) !}\right]^{2} .
\end{array}
$$

\footnotetext{
${ }^{*}$ Received July 28, 1983. The publication of this note was supported by the Applied Research Laboratory of the Pennsylvania State University under contract with the Naval Sea Systems Command.
} 
Suppose that one now translates each of the spherical wave functions in the summation from the primed coordinates back to the unprimed coordinates. The transformation to accomplish this differs in form from Eq. (1) only in that an additional factor of $(-1)^{\nu}$ appears in the summand to account for the reversal in the direction of translation. One now obtains a representation of the original $n$th order spherical wave function that appears to involve a doubly infinite summation over all possible orders of the same functions. Naturally, the coefficient of every order wave function except the $n$th order must be zero while that of the $n$th order term must be unity. These coefficients are readily seen to be an infinite series of spherical Bessel functions of argument $k r_{0}$. Since the distance $r_{0}$ is arbitrary, these series must converge to either zero or unity for all $k r_{0}$. Following this reasoning, a collection of infinite series involving spherical Bessel functions has been obtained; most of these series are believed to be novel.

As a specific example, consider the zero order spherical wave function. From Eq. (1),

$$
h_{0}(k r)=\sum_{m=0}^{\infty}(-1)^{m}(2 m+1) j_{m}\left(k r_{0}\right) h_{m}\left(k r^{\prime}\right) P_{m}\left(\cos \theta^{\prime}\right) \text {. }
$$

Shifting each wave function, $h_{m}\left(k r^{\prime}\right) P_{m}\left(\cos \theta^{\prime}\right)$, back to origin 0 produces

$$
\begin{gathered}
h_{0}(k r)=\sum_{m=0}^{\infty} \sum_{\nu=0}^{\infty} \sum_{p} i^{m+p-\nu}(2 m+1)(2 \nu+1) a(0,0 \mid p, \nu, m) \\
\cdot j_{m}\left(k r_{0}\right) j_{\nu}\left(k r_{0}\right) h_{p}(k r) P_{p}(\cos \theta) .
\end{gathered}
$$

From Eq. (2), it is evident that index $p$ can only equal zero if $\nu=m$. Therefore, the coefficient of $h_{0}(k r)$ from the right hand side of Eq. (5) is

$$
\sum_{m=0}^{\infty}(2 m+1)^{2} a(0,0 \mid 0, m, m) j_{m}^{2}\left(k r_{0}\right)=1
$$

Since, from Eq. (3) $a(0,0 \mid 0, m, m)=(2 m+1)^{-1}$, Eq. (6) becomes (with $k r_{0}=\xi$ )

$$
\sum_{m=0}^{\infty}(2 m+1) j_{m}^{2}(\xi)=1, \quad \text { for all } \xi
$$

which is a documented result, [3]. The coefficient of $h_{p}(k r) P_{p}(\cos \theta)$ for any $p>0$ must vanish. From Eq. (2), the index $p$ can equal unity only for these conditions: $\nu=m+1$ for $m \geqslant 0$ and $\nu=m-1$ for $m \geqslant 1$. Hence, from Eqs. (2) and (5), one obtains the following result corresponding to $p=1$

$$
\sum_{m=0}^{\infty}\left[(m+1) j_{m+1}(\xi)-m k_{m-1}(\xi)\right] j_{m}(\xi)=0 .
$$

This can readily be reduced to a trivial identity by shifting the index of either of the two terms; note, however, that by use of a standard recurrence relationship for $j_{m}^{\prime}(\xi)$, the derivative of $j_{m}(\xi)$ with respect to its argument, Eq. (8) can also be written

$$
\sum_{m=0}^{\infty}(2 m+1) j_{m}(\xi) j_{m}^{\prime}(\xi)=0
$$


Proceeding in a similar manner, the coefficients of higher order spherical wave functions from the right-hand side of Eq. (5) were developed. The resulting series of spherical Bessel functions, which become more complicated as the mode order increases, must also equal zero.

Additional series were then developed by considering the translation to origin $0^{\prime}$ and back to 0 of the first five orders of wave functions. As might be anticipated, the coefficient of the $m$ th order wave function in the doubly infinite series representation of the $n$th mode was, within a constant multiplicative factor, the same infinite series of spherical Bessel functions as the coefficient of the $n$th order wave function in the series representation of the $m$ th mode; these series must necessarily sum to zero. The series that must sum to unity was, however, different for each different mode considered.

The results that were generated, by considering this double shift of the first five spherical wave functions, and by developing the coefficients of the first five wave functions in the doubly infinite series representation, are collected in Tables I and II. Note that while the term $A_{n}$ is proportional to just $j_{n}(\xi)$ and $B_{n}$ is proportional to just $j_{n}^{\prime}(\xi), C_{n}$ is not proportional to just $b_{n}^{\prime \prime}(\xi)$ nor $D_{n}$ to just $j_{n}^{\prime \prime \prime}(\xi)$, etc.

\section{TABLE I}

A Collection of Series Involving Spherical Bessel Functions

(term defined in Table II)

(a) Series that sum to unity for any argument, $\xi$

$$
\begin{gathered}
\sum_{n=0}^{\infty} \frac{1}{(2 n+1)} A_{n}^{2}(\xi)=1 \\
3 \sum_{n=0}^{\infty} \frac{1}{(2 n+1)} B_{n}^{2}(\xi)=1 \\
5 \sum_{n=0}^{\infty} \frac{1}{(2 n+1)} C_{n}^{2}(\xi)=1 \\
7 \sum_{n=0}^{\infty} \frac{1}{(2 n+1)} D_{n}^{2}(\xi)=1 \\
9 \sum_{n=0}^{\infty} \frac{1}{(2 n+1)} E_{n}^{2}(\xi)=1
\end{gathered}
$$

(b) Series that sum to zero for any argument, $\xi$

$$
\sum_{n=0}^{\infty} \frac{1}{(2 n+1)} \alpha_{n}(\xi) \beta_{n}(\xi)=0
$$

where $\alpha_{n}(\xi)$ and $\beta_{n}(\xi)$ are any two different terms from Table II. 


\section{TABLE II}

\section{Terms for the Series in Table I}

$$
\begin{aligned}
& A_{n}(\xi)=(2 n+1) j_{n}(\xi) \\
& B_{n}(\xi)=(2 n+1) j_{n}^{\prime}(\xi)=n j_{n-1}(\xi)-(n+1) j_{n+1}(\xi) \\
& C_{n}(\xi)=\frac{3(n-1) n}{2(2 n-1)} j_{n-2}(\xi)-\frac{n(n+1)(2 n+1)}{(2 n-1)(2 n+3)} j_{n}(\xi)+\frac{3(n+1)(n+2)}{2(2 n+3)} j_{n+2}(\xi) \\
& D_{n}(\xi)=\frac{5(n-2)(n-1) n}{2(2 n-3)(2 n-1)} j_{n-3}(\xi)-\frac{3(n-1) n(n+1)}{2(2 n-3)(2 n+3)} j_{n-1}(\xi) \\
& \quad+\frac{3 n(n+1)(n+2)}{2(2 n-1)(2 n+5)} j_{n+1}(\xi)-\frac{5(n+1)(n+2)(n+3)}{2(2 n+3)(2 n+5)} j_{n+3}(\xi) \\
& E_{n}(\xi)=\frac{35(n-3)(n-2)(n-1) n}{8(2 n-5)(2 n-3)(2 n-1)} j_{n-4}(\xi)-\frac{5(n-2)(n-1) n(n+1)}{2(2 n-5)(2 n-1)(2 n+3)} j_{n-2}(\xi) \\
& +\frac{9(n-1) n(n+1)(n+2)(2 n+1)}{4(2 n-3)(2 n-1)(2 n+3)(2 n+5)} j_{n}(\xi)-\frac{5 n(n+1)(n+2)(n+3)}{2(2 n-1)(2 n+3)(2 n+7)} j_{n+2}(\xi) \\
& +\frac{35(n+1)(n+2)(n+3)(n+4)}{8(2 n+3)(2 n+5)(2 n+7)} j_{n+4}(\xi)
\end{aligned}
$$

\section{REFERENCES}

[1] S. Stein, Addition theorems for spherical wave functions, Quart. Appl. Math. 19 15-24 (1961)

[2] W. Thompson, Jr., Acoustic radiation and scattering from two eccentrically positioned spheres, Ph.D. dissertation, The Pennsylvania State University, 1971

[3] M. Abramowitz and I. A. Stegun, Handbook of Mathematical Functions, Dover Publications, New York 440, 1965 